\title{
Universiteit
}

Leiden

The Netherlands

\section{Shock waves in two-dimensional granular flow: Effects of rough walls and polydispersity}

Hørlück, S.; Hecke, M.L. van; Dimon, P.

\section{Citation}

Hørlück, S., Hecke, M. L. van, \& Dimon, P. (2003). Shock waves in two-dimensional granular flow: Effects of rough walls and polydispersity. Physical Review E, 67(2), 021304. doi:10.1103/PhysRevE.67.021304

Version: $\quad$ Publisher's Version

License: $\quad$ Leiden University Non-exclusive license

Downloaded from: https://hdl.handle.net/1887/81225

Note: To cite this publication please use the final published version (if applicable). 


\title{
Shock waves in two-dimensional granular flow: Effects of rough walls and polydispersity
}

\author{
Sune Hørlück, Martin van Hecke, ${ }^{*}$ and P. Dimon \\ The Center for Chaos and Turbulence Studies, The Niels Bohr Institute, Blegdamsvej 17, DK-2100 Copenhagen 0 , Denmark
}

(Received 27 September 2002; published 26 February 2003)

\begin{abstract}
We have studied the two-dimensional flow of balls in a small-angle funnel, when either the side walls are rough or the balls are polydisperse. As in earlier work on monodisperse flows in smooth funnels, we observe the formation of kinematic shock waves (density waves). We find that for rough walls the flows are more disordered than for smooth walls and that shock waves generally propagate more slowly. For rough wall funnel flow, we show that the shock velocity and frequency obey simple scaling laws. These scaling laws are consistent with those found for smooth wall flow, but here they are cleaner since there are fewer packing-site effects and we study a wider range of parameters. For pipe flow (parallel side walls), rough walls support many shock waves, while smooth walls exhibit fewer or no shock waves. For funnel flows of balls with varying sizes, we find that flows with weak polydispersity behave qualitatively similar to monodisperse flows. For strong polydispersity, scaling breaks down and the shock waves consist of extended areas where the funnel is blocked completely.
\end{abstract}

DOI: 10.1103/PhysRevE.67.021304

PACS number(s): 45.70.Mg, 45.70.Vn

\section{INTRODUCTION}

Density waves can occur in various granular flow systems such as funnels and hoppers [1], pipes [2], and hour glasses [3]. In previous experiments, we studied the formation of kinematic shock waves which propagate against the main flow in a two-dimensional system of balls rolling in a smallangle funnel [4-6]. A sketch of the setup used is shown in Figs. 1(a,b). In this earlier work, the walls of the funnel were smooth, and the balls were of equal size (monodisperse flow). In the present work, we study what happens when we break the peculiarities of this smooth-wall-monodisperse system by either (i) making the walls of the funnel rough [Fig. 1(c)], or (ii) taking "polydisperse" mixtures of balls of different sizes [Fig. 1(d)].

The crucial experimental parameters characterizing the flow geometry are the funnel opening angle $\beta$ and the funnel outlet width $D$. For smooth-wall-monodisperse flows the most important features of the shock waves were found to be the following [4-6]: (i) For $\beta>0$, the rolling grains tend to locally form triangular lattices which lead to the creation of shock waves predominantly at particular sites in the funnel where close packing occurs. (ii) The velocities $U$ of the shocks are, in good approximation, a function of the rescaled width $w(x) / D$ only. Here $x$ is the coordinate along the funnel [Fig. 1(b)] and $w(x)$ denotes the funnel width at position $x$.

As we will show below, by making the walls rough or the flow polydisperse, the triangular packing can (partially) be suppressed. We have studied the shock statistics and the behavior of individual balls as in previous work [5,6], and will present here the results for the creation and propagation of shocks in these systems in the pipe flow (parallel walls) and in the small angle, intermittent flow regime $[5,6]$. We have also investigated, using ball tracking, the formation of shear

\footnotetext{
*Present address: Kamerlingh Onnes Lab, Universiteit Leiden, P.O. Box 9504, 2300 RA Leiden, The Netherlands.
}

bands for rough walls and the effects of completely stationary shock packings in polydisperse flows.

Recently, a number of related experiments on twodimensional flow have been performed. Tsai et al. [7] studied a system close to our rough wall pipe flow experiment, and included the effect of partially blocking the outlet. Reydellet, Rioual, and Clément [8] studied a falling vertical column of balls, where ball-ball interactions are dominated by collisions, and the rolling of balls does play a minor role. Finally, Le Pennec et al. [9] studied two-dimensional rolling flows of small glass balls in flow geometries with very large funnel angles.

The experimental setup and methods of analysis have been described in detail elsewhere $[5,6]$ and we summarize the essential aspects here. A sketch of the setup is shown in Fig. 1(a) and the important geometric parameters are shown
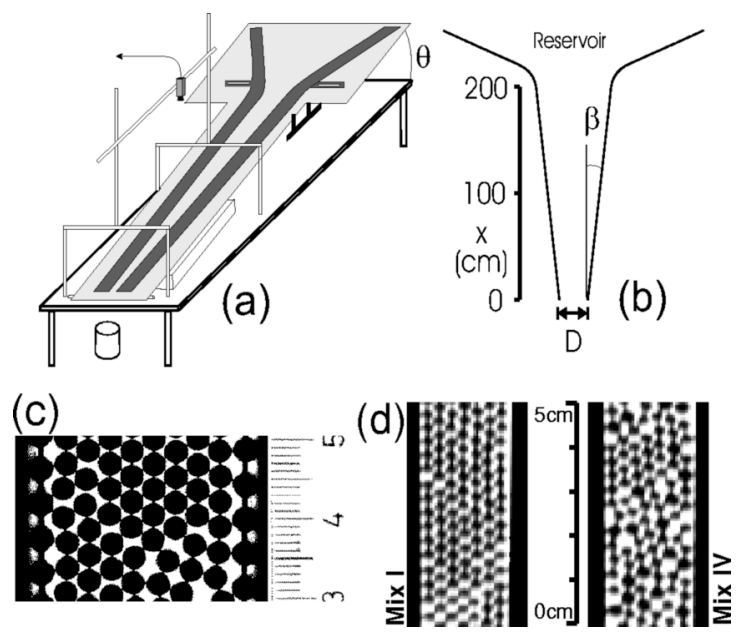

FIG. 1. (a) Sketch of the experimental setup. (b) Schematic top view of the setup, showing the important parameters of the funnel geometry. (c) Closeup of the balls rolling between rough walls. The linked balls near the edges are glued to the walls (the scale is in $\mathrm{cm}$ ). (d) Snapshots of two different polydisperse mixtures, referred to as Mix I and Mix IV (see text). 


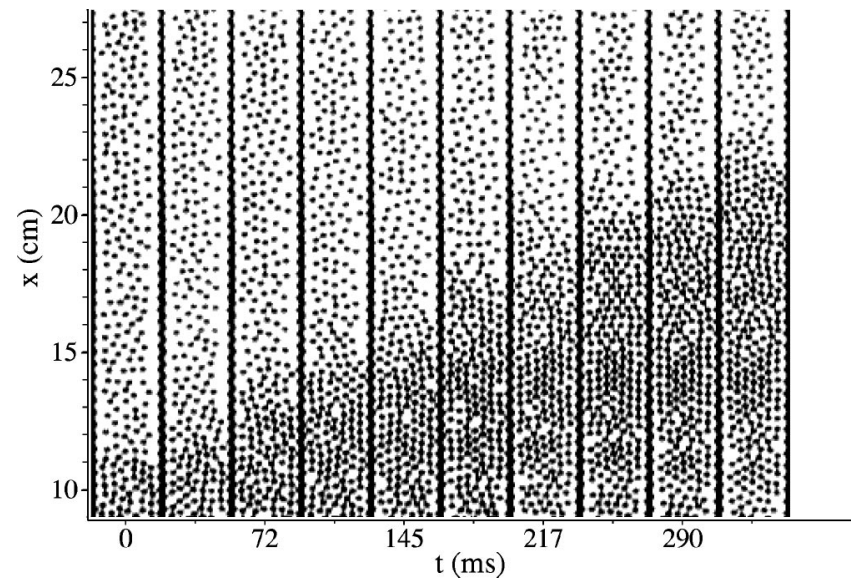

FIG. 2. Film sequence showing $18 \mathrm{~cm}$ of the funnel showing a propagating shock wave at $\beta=0.2^{\circ}, D=20 \mathrm{~mm}$ (each vertical "stripe" is a separate picture). Such movies are recorded at 220 frames/s and are used for the ball tracking discussed in Sec. II C. For clarity, only every eight frame is shown.

in Fig. 1(b). The balls roll on a coated Lexan plane (with inclination $\theta=4.1^{\circ}$ ) in a single layer, in a funnel formed by aluminum walls and covered by a transparent lid. The granular material is comprised of 50000 brass balls of $3.18 \mathrm{~mm}$ diameter in the monodisperse case, while details of the polydisperse mixtures are discussed in Sec. III. The rough walls were made by glueing linked rows of balls of nearly the same diameter as the rolling balls to the original smooth walls [Fig. 1(c)]. The walls are straight for $200 \mathrm{~cm}$ and curve smoothly at the top to form a reservoir. They can be moved to vary the outlet width $D(0-35 \mathrm{~mm})$ and the funnel half angle $\beta\left(0^{\circ}-3^{\circ}\right)$. A light box is placed below the funnel to illuminate the balls from below, and a video camera is placed above the system. Snapshots of a small part of the funnel show the effect of rough walls [Fig. 1(c)] and polydispersity [Fig. 1(d)] on the packing of the balls.

The paper is structured as follows. In Sec. II we present data based on monodisperse flows between rough walls. Shock wave statistics for both intermittent flows $\left(\beta>0^{\circ}\right)$ and for pipe flows $\left(\beta=0^{\circ}\right)$ are discussed, and we also study data based on ball tracking. In Sec. III we investigate the effect of various degrees of polydispersity in flow between smooth walls. Finally, in Appendix A, we discuss some similarities with traffic flow.

\section{ROUGH WALLS}

The basic phenomenology of the formation of shock waves is illustrated in Fig. 2, which shows ten subsequent snapshots of the balls in a small section near the outlet of the rough wall funnel. In dense regions, which may occur due to a combination of geometric effects (the finite funnel angle $\beta$ ) and the inelastic nature of the collisions, kinetic energy is dissipated rapidly and so the balls here have a lower velocity than the balls in dilute regions. This leads to kinematic shock waves, in which the balls become almost stationary and tend to pack in a lattice which extends from wall to wall. In such a region, both energy and momentum are efficiently trans-
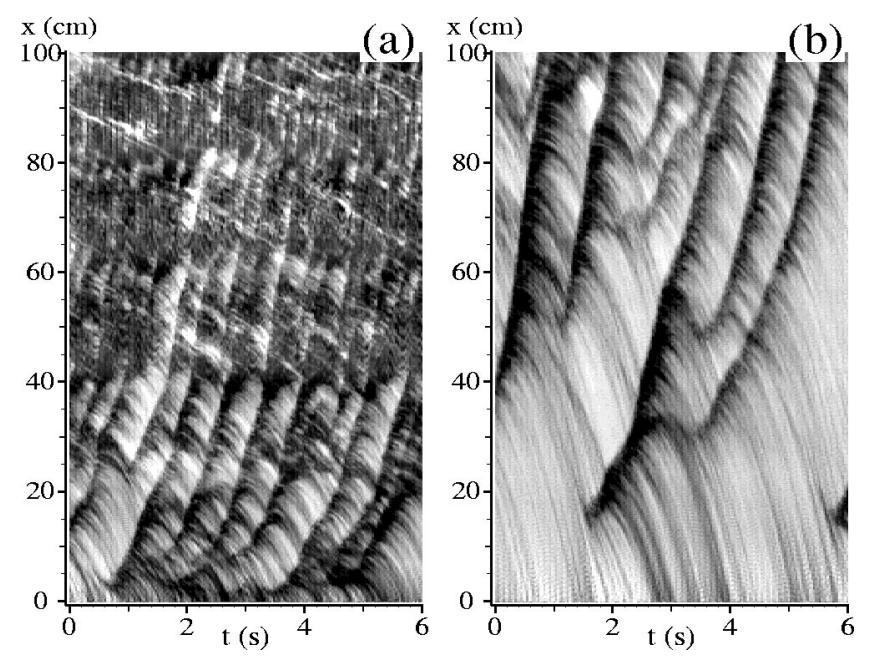

FIG. 3. Space-time plots of the density $\tilde{\rho}(x, t)$ at $\beta=0.4^{\circ}$ for (a) $D=15 \mathrm{~mm}$ and (b) $D=25 \mathrm{~mm}$.

ferred to the walls. Shocks grow in the upstream direction due to incoming, high velocity balls, and dissolve in the downstream direction due to the action of the (effective) gravity, which eventually accelerates the balls again. To analyze these shocks, we recorded $\sim 2-9 \mathrm{~s}$ movies of $37 \mathrm{~cm}$ sections of the flow (usually at 220 frames/s), and applied ball tracking software [6], gaining detailed knowledge of the position, velocity and acceleration of the individual balls (see Sec. II C).

In many cases, however, one may be more interested in the overall features of the shocks and not so much in the individual balls. For such analysis, sequences of images taken at 60 frames/s and covering $100 \mathrm{~cm}$ sections of the funnel were averaged in the transverse direction to obtain a one-dimensional relative density profile. An example of a space-time plot of this relative density $\tilde{\rho}(x, t)$ is shown in Fig. 3 for two different sets of parameters. From these the position of a shock wave and its creation site, average local velocity $U(x)$ and frequency $\nu(x)$ can be determined. As with smooth walls, shock waves between rough walls are easily distinguished by eye [5]. They are created at various positions in the funnel and there are some noticeable interactions between shocks. As in earlier work on smooth walls, shock waves are created more often at large funnel angles and for small values of $D$. The shock velocity $U$ is generally observed to grow with increasing $\beta, x$, and decreasing $D$.

\section{A. $\boldsymbol{\beta}>\mathbf{0}$}

We will study now in detail the statistics of shock creation, velocity, and frequency, based on density data taken for $D$ ranging from 15 to $30 \mathrm{~mm}$, and for $\beta$ ranging from $0.1^{\circ}$ up to $1.2^{\circ}$. The case of pipe flow $(\beta=0)$, where rough and smooth walls show very different behavior, will be studied in Sec. II B, and some results following from ball tracking are discussed in Sec. II C.

\section{Shock creation}

The monodispersity of the balls permits them to form close packed triangular lattices at certain packing sites $x$ $=\chi_{i}$ in the funnel. For smooth walls, the positions of these packing sites are given by 


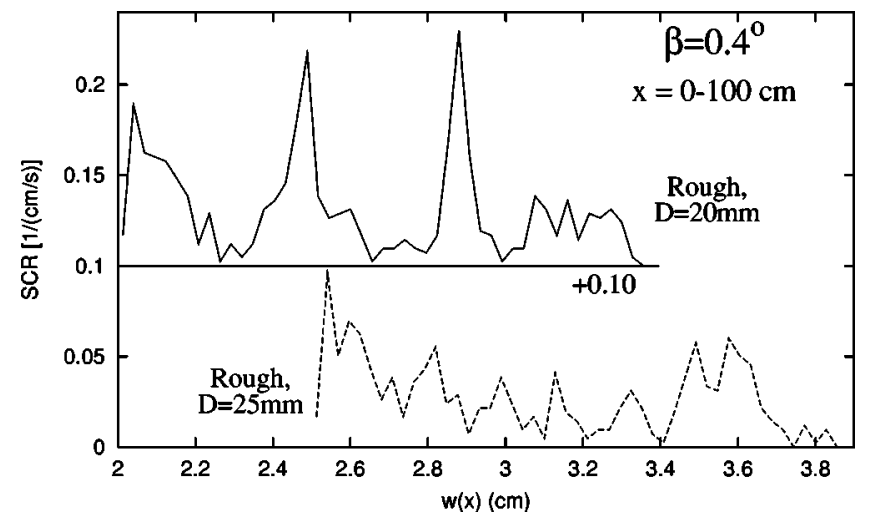

FIG. 4. Manually counted shock creation rates (SCR) [13] for rough walls shown as a function of the local funnel width $w(x)$ for $D=20 \mathrm{~mm}$ and $D=25 \mathrm{~mm}$ (both data sets cover $x=0-100 \mathrm{~cm}$ ).

$$
\chi_{i}=\frac{2 r+\sqrt{3} r(i-1)-D}{2 \tan \beta}
$$

where $i$ is an integer and $r$ is the ball radius [5]. In a funnel with smooth walls, packing effects are rather pronounced as shown in Fig. 18 of Ref. [5].

For rough walls, it is slightly more difficult to give an estimate of where one expects packing sites. It is, for example, not obvious whether the value of $D$ (which is the minimum distance between the two rough walls) is the relevant parameter. We have found that packing effects persist from small $D$, but are washed out for larger funnel outlets. This is illustrated in Fig. 4, which shows the manually counted shock creation rates for two different values of $D$ as a function of the local width $w$ (this aligns possible packing sites). Clearly, packing effects are present for $D=20 \mathrm{~mm}$, but are washed out for $D=25 \mathrm{~mm}$. This is consisted with the $D=15 \mathrm{~mm}$ and $D=30 \mathrm{~mm}$ data sets, although in these cases it is difficult to obtain a precise estimate for the shock creation rates. A comparison of the data shown in Fig. 4 to data on the shock creation rates in smooth funnels, as shown in the top of Fig. 12, confirms that rough walls suppress the effects of packing.

We therefore have shown that packing effects are suppressed in sufficiently wide rough wall flow, and that in this case shock waves are created everywhere in the funnel with equal probability.

\section{Shock velocity}

From the density fields, we can obtain the local shock velocity $U(x)$ using the shock detection algorithm RGE (Relative density contrast Gradient Edge detection) [11]. This is a refinement of the GE-method described in detail in Ref. [5], and is used to measure $U(x)$ and $\nu(x)$. The shock velocities obtained with this method for forty different sets of parameters are shown in Fig. 5(a) as a function of the local funnel width $w(x)$. This already indicates a clustering of the data in groups given by the funnel outlet $D$. When the data is replotted against the dimensionless parameter $w(x) / D$ as in Fig. 5(b), there is a fairly good data collapse with $U(x) \approx 79[w(x) / D-0.73] \mathrm{cm} / \mathrm{s}$. A similar scaling
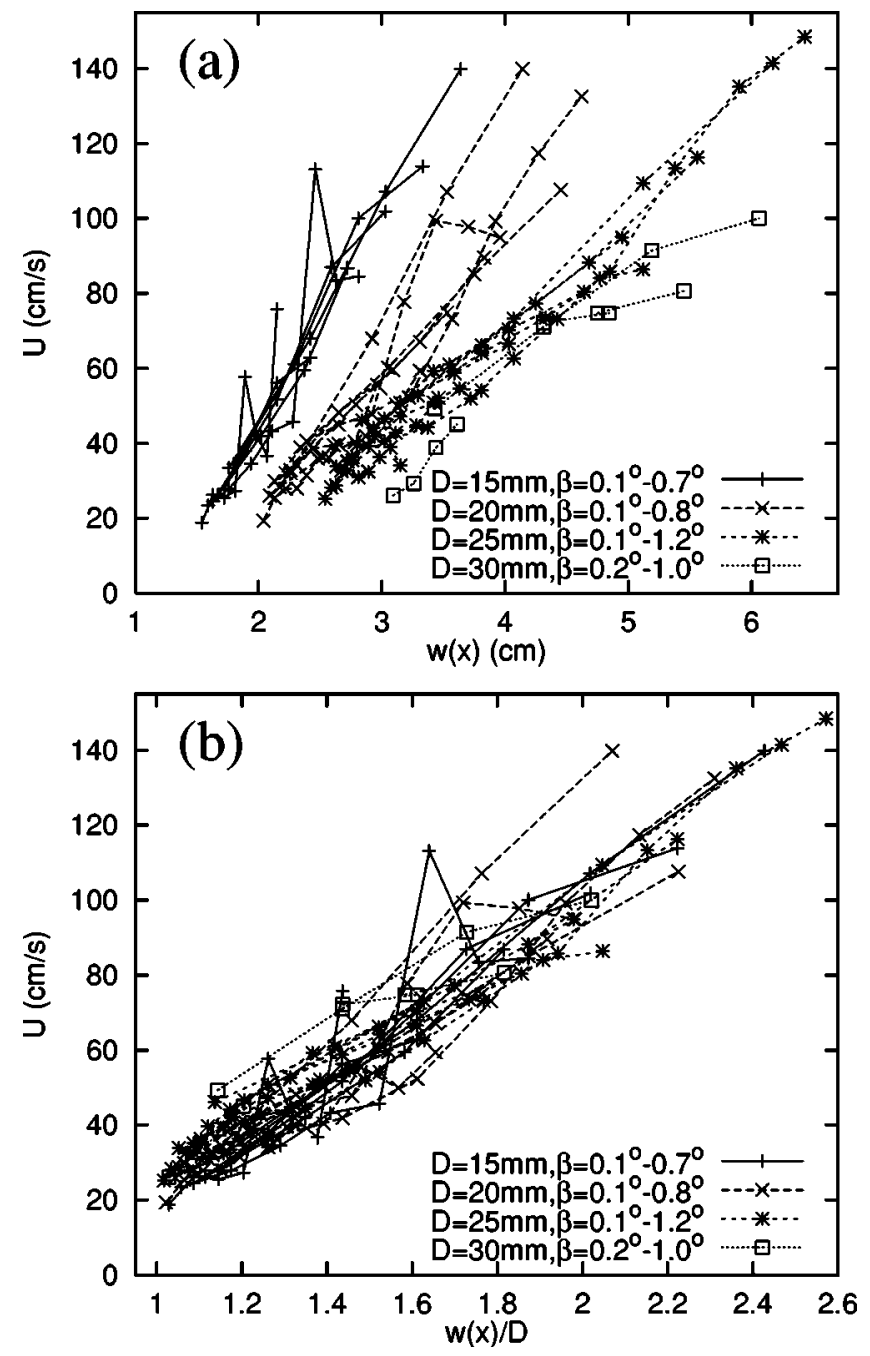

FIG. 5. Average shock velocity $U(x)$ for 40 different sets of parameters $\beta$ and $D$ as function of (a) the local width $w(x)$ and (b) the rescaled, nondimensionalized width $w(x) / D$.

$[U(x) \approx 64[w(x) / D-0.14] \mathrm{cm} / \mathrm{s}]$ was observed for smooth walls $[5,6]$. Experiments with one rough and one smooth wall, which we will refer to as "semi rough" (not shown) gave a more disordered flow with a decent data collapse of $U(x) \approx 83[w(x) / D-0.57] \mathrm{cm} / \mathrm{s}$, which falls between rough wall and smooth wall data for low $w(x) / D$ [see Fig. 7(a)]. Therefore we conclude that the roughness of the walls leads, in general, to a slowing down of the shock waves.

In the data collapse Fig. 5(b) there seems to be a trend for wide funnels to have slightly larger velocities. Some of the $D=30 \mathrm{~mm}$ data sets had problems with static buildup at the outlet during parts of the experiment, which is likely to have affected shock statistics [increasing $U(x)$ and $\nu(x)$ ] near the outlet. It is also possible that for larger outlet widths the data for rough walls becomes more comparable to those of smooth wall systems. Nevertheless, we find that the shock velocity is in good approximation a function of the local rescaled width $w(x) / D$ only, both for smooth and rough walls. 


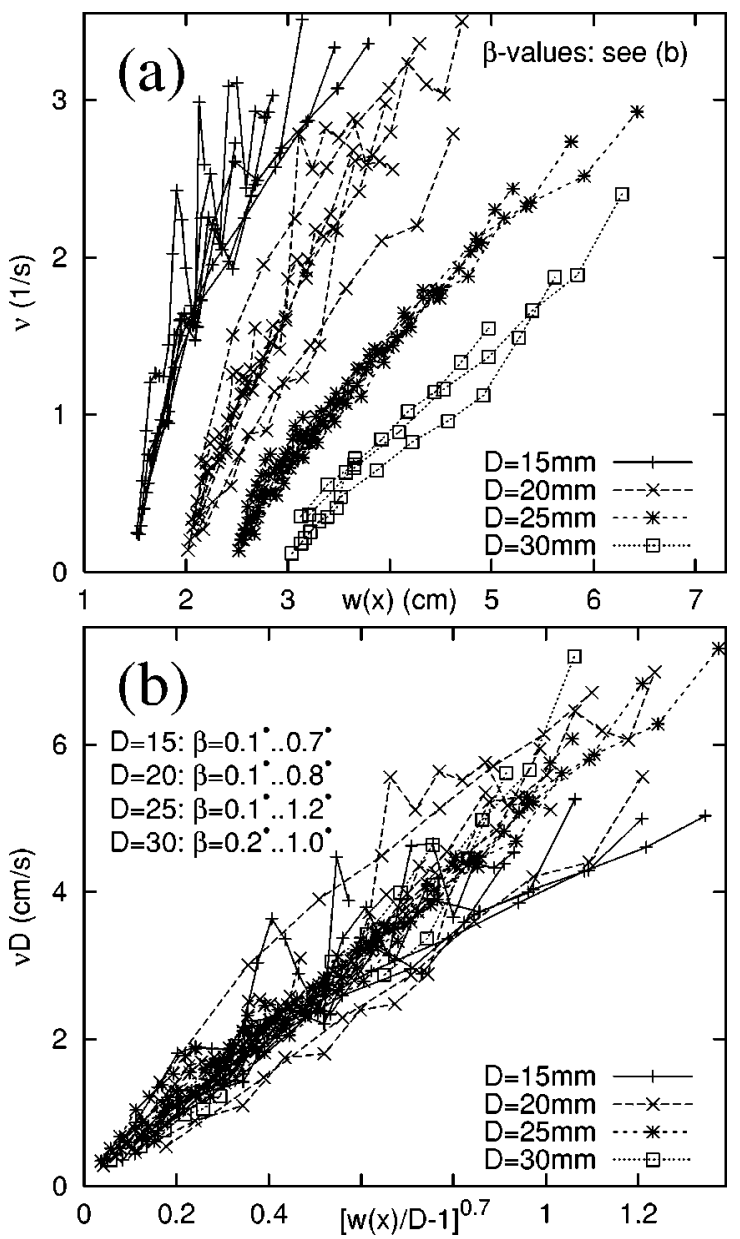

FIG. 6. (a) Average shock frequency $\nu(x)$ vs $w(x)$. (b) Rescaled shock frequency $\nu(x) / D$ vs $[w(x) / D-1]^{0.7}$ (lin/lin). All for 40 different sets of parameters $\beta$ and $D$.

\section{Shock frequency}

From the density plots we can also obtain the local shock frequencies $\nu(x)$ using the RGE algorithm [11]. In Fig. 6(a) we show the shock frequencies for our rough wall data as a function of $w(x)$. While some packing site periodicity is still visible for $D=15 \mathrm{~mm}$, for larger values of $D$ the curves are fairly smooth and we will discuss the data collapse that occurs there.

From the data shown in Fig. 6(a) it appears that $\nu$ goes to zero when $w(x) \rightarrow D$, i.e., near the outlet. Replotting the data then as function of $w(x) / D-1$ (not shown) confirmed this, and indicated that to achieve data collapse, one needs to plot the product $\nu(x) D$ as a function of $w(x) / D-1$. Finally, in contrast to the shock velocity, the frequency clearly grows nonlinearly with $w(x) / D-1$. On a log-log plot, it appears that $\nu D$ scales as $[w(x) / D-1]^{p}$, with an exponent $p$ around 0.7 [Fig. 6(b)]. While the scaling range is to small to determine whether such power law scaling holds asymptotically, it is a useful way of collapsing our data, as also is shown in Fig. 6(c). Note that significant deviations can be seen for larger values of $w(x) / D$. The data falling below the curve in Fig. 6(c) mainly belong to data sets with $D=15 \mathrm{~mm} \beta$
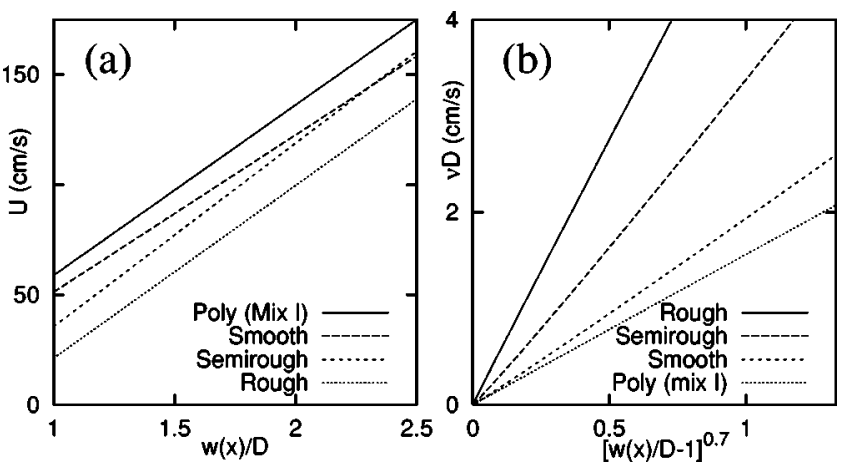

FIG. 7. Linear fits for $1 \leqslant w(x) / D \leqslant 2.5$ of $U(x)$ vs $w(x) / D$ in (a) and for $\nu(x) D$ vs $w(x) / D$ in (b). Both graphs show the lines for smooth wall, smooth or rough combined walls and rough wall flows. Data for weakly polydisperse, smooth-wall flows are also included.

$\geqslant 0.5^{\circ}$, where the RGE method tends to underestimate $\nu(x)$ for large $x, \beta$ [11].

In a previous paper [5], which discussed the shock dynamics for smooth funnels, the issue of $\nu(x)$ data collapse was not discussed [12]. Reprocessing of this smooth-wall data employing the RGE algorithm does show a decent data collapse, where $\nu(x) D=1.9(1)[w(x) / D-1]^{0.7(1)}$, but the number of old data sets with $D \neq 10 \mathrm{~mm}$ that gives reliable $\nu(x)$ data is insufficient to judge whether $\nu(x) D$ or $\nu(x)$ gives a better data collapse. We will discuss this issue below for weakly polydisperse mixtures in smooth funnels in Sec. III 2, where more data is available.

Note that the shock frequency for smooth walls is generally less than half the frequency for rough walls. However, since we believe that shock waves are, in general, produced by disturbances in the flow, this should not be a big surprise: rough walls produce more disturbances.

Data obtained from flows with one rough wall and one smooth wall ("semi rough") confirm this intuitive picture, since we find a decent data collapse of $\nu(x) D$ $\approx 3.4(1)[w(x) / D-1]^{0.7(1)} \mathrm{cm} / \mathrm{s}$ (not shown). This is right between the fits for the rough wall and smooth-wall data discussed above.

\section{Rough wall intermittent flow: Conclusion}

The rough walls allow us to suppress packing effects, so that two simple scaling laws for the shock velocity and frequency emerge:

$$
\begin{gathered}
U(x) \approx \alpha_{1}\left[w(x) / D-\alpha_{2}\right], \\
\nu(x) \approx D^{-1} \alpha_{3}[w(x) / D-1]^{0.7} .
\end{gathered}
$$

The fitting coefficients $\alpha_{1}, \alpha_{2}$ and $\alpha_{3}$ vary with the roughness of the walls. In Fig. 7 we show the best fits for data obtained for rough walls, semirough walls, smooth-walls, and smooth-walls-weakly polydisperse balls (discussed in detail in Sec. III 2). The fits are all made in the interval 1 $\leqslant w(x) / D \leqslant 2.5$ for consistency.

As shown in Fig. 7(a), changes in the boundaries have a weak effect on $\alpha_{1}$, but substantially affect $\alpha_{2}$. The old 
smooth-wall data for the velocity seems to deviate a little from this trend, and we do not know the reason for this. For the frequency, the roughness of the walls has a profound influence on the prefactor $\alpha_{3}$. For small-angle funnels, rough walls promote the occurrence of shock waves, but do not affect their propagation velocity substantially.

\section{B. Pipe flow}

The behavior of shocks in pipe flow $\left(\beta=0^{\circ}\right)$ can be expected to be rather different from those in intermittent flow $\left(\beta \geqslant 0.1^{\circ}\right)$ since for pipe flow there is no "geometric" source for the formation of shock waves, and dissipative collisions are now presumably the dominant origin of shock wave formation.

Indeed, for smooth walls the (few) shock waves that are generated never reach the reservoir and consequently the flow rate (and indirectly all other flow properties) are entirely determined by the reservoir outflow $[4,5]$. Surprisingly, the qualitative features of pipe flow with rough walls are similar to the intermittent flows. For example, many shocks do travel upwards and reach the reservoir.

Similar to smooth-wall pipe flow, the rough wall pipe flow is extremely sensitive to exact experimental conditions and consequently the statistics obtained for these flows is bound to be more noisy. In fact, as we will see below, subtle problems near the outflow area render part of the data unusable. The analysis of shock properties is further hampered by the fact that the RGE shock identification method [11] used above has problems detecting some shocks with $U<15$ $-20 \mathrm{~cm} / \mathrm{s}$. We have therefore decided to use an alternative method, based on space-time correlation functions, to obtain a measure of the velocity, and determine their frequency based on a simple threshold algorithm (see Appendix B).

Based on these methods, we have determined the shock velocities for rough wall pipe flow. We find that the data for the lower part of the funnel $(0<x<100 \mathrm{~cm})$ does not show any systematic trend, and often here data sets show a complicated mixture of periods of stationary shocks and moving shocks. We think that this is due to experimental problems (in particular static charge buildup near the funnel outlet in some measurements). In later runs, when we studied the upper funnel $(100<x<200 \mathrm{~cm})$, these problems were solved. Here we find that the shock velocity is essentially independent of the width $D$, apart from some effects near the outflow of the reservoir [see Fig. 8(a)].

The shock frequencies decrease with $D$, but do not show a clear trend with $x$. Rescaling the frequency with $D$, we find that $\nu D$ is fairly constant, for $D$ ranging from $15 \mathrm{~mm}$ to 30 $\mathrm{mm}$ [see Fig. 8(b)]. Again, most data for $(0<x<100 \mathrm{~cm})$ does not show any systematic behavior, due to the large amount of stationary shocks.

Using the linear fits of $U(x)$ and $\nu(x) D$ similar to Sec. II A, we find $U(w(x)=D) \approx 21 \mathrm{~cm} / \mathrm{s}$ and $\nu[w(x)=D] D$ $\approx 0.8 \mathrm{~cm} / \mathrm{s}$. Considering the systematic differences between RGE based and space-time correlation based measurements of $U(x)$ there is a fairly good correspondence between the pipe flow shock wave data from the $x>100 \mathrm{~cm}$ part of the funnel and the shock wave behavior for $\beta>0^{\circ}$ flows. This
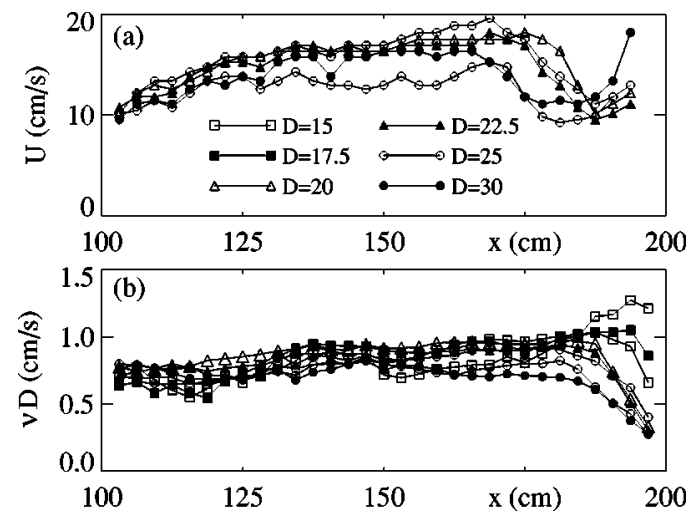

FIG. 8. Velocity (a) and rescaled frequency (b) of shock waves in rough wall pipe flow.

suggests that the mechanisms of shock wave propagation are not too different after all.

We conclude that in good approximation, rough wall shock flow is dominated by shocks that are created mainly near the outflow region, travel upward with fairly constant velocity and whose frequency decreases as $1 / D$.

\section{Ball tracking}

We have processed detailed films such as shown in Fig. 2 to obtain ball trajectories (details of this method are described in Ref. [6]). From the full set of ball trajectories, we have constructed continuous one-dimensional Eulerian fields of the relative density $\tilde{\rho}(x, t)$, velocity $v(x, t)$, and acceleration $a(x, t)[6]$. A comparison of the acceleration fields for rough and smooth walls indicates that the flow for smooth walls has more disturbances and the shocks are less sharply defined.

We have previously shown (Appendix C in Ref. [6]) that smooth-wall flows are reasonably one dimensional, i.e., the density, velocity and acceleration fields do not show a strong dependence on $y$, the coordinate across the funnel. This is no longer true for rough wall flows as shown in Fig. 9. The velocity $\left\langle v_{x}\right\rangle$ as a function of the transverse coordinate $y$ is shown for various pipe flows in Fig. 9(a) and for $\beta>0^{\circ}$ flows in Fig. 9(b). In both cases do we find that $\left\langle v_{x}\right\rangle$ drops off near the boundaries, and this effect is most pronounced for pipe flow and small-angle flow. A number of different flow behavior could underly these statistics, the most obvious being: (i) The flow has a shear component near the walls, i.e., balls near walls move typically slower than in the bulk. (ii) In shock packings the flow reaches from wall to wall, but in fast regions (between shocks) balls near walls are repulsed. In this interpretation, balls in fast regions are repelled from the boundaries, and this transverse momentum is absorbed via ball-ball interactions in the funnel center, leaving the regions near the walls relatively empty.

To resolve this ambiguity we plot histograms of the $y$ positions of balls with their $v_{x}$ in a certain interval in Fig. 9(c) (for pipe flow) and in Fig. 9(d) (for $\beta=0.4^{\circ}$ flow). If reason (ii) would dominate, the histogram of the slowest balls (in shock regions) should be fairly flat, but this is not 

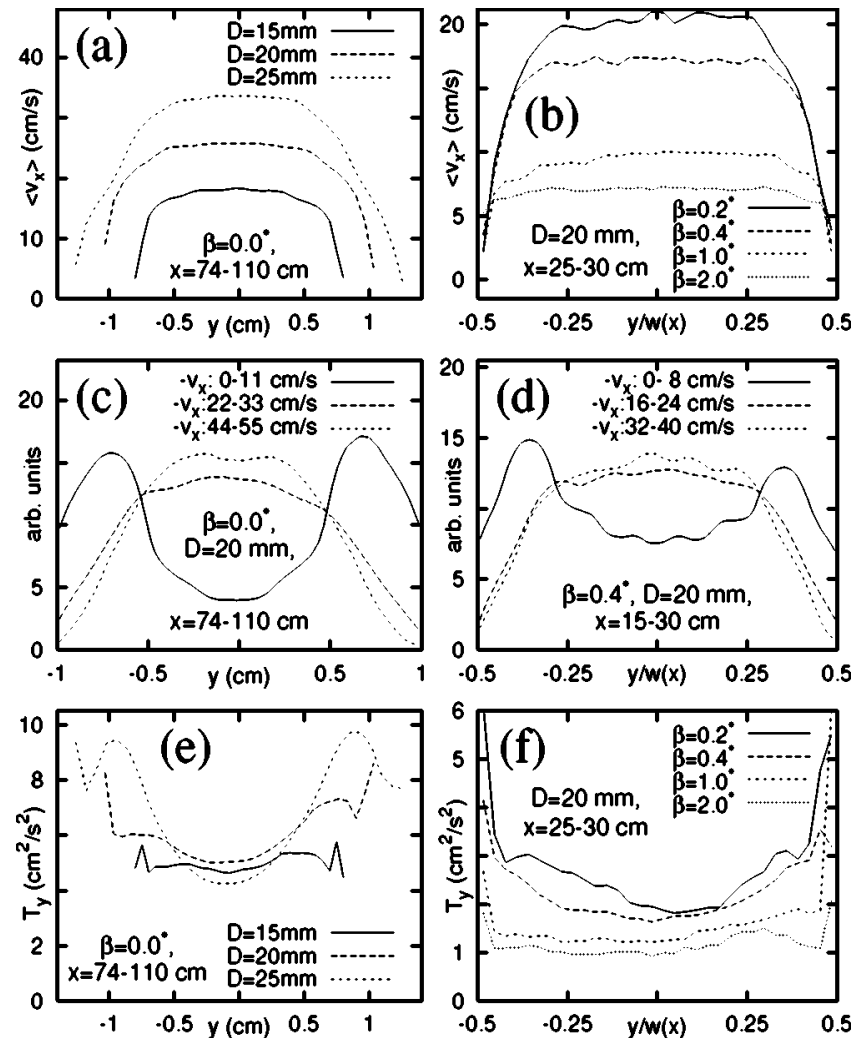

FIG. 9. Average $v_{x}$ as function of $y$ in various pipe flows (a) and $\beta \geqslant 0.1^{\circ}$ flows (b). Histograms of ball positions (considering the width of the balls) grouped according to the $v_{x}$ value for a pipe flow $\left(\beta=0.0^{\circ}, D=20 \mathrm{~mm}\right)$ in (c) and intermittent flow $\left(\beta=0.4^{\circ}, D\right.$ $=20 \mathrm{~mm}$ ) in (d). The average square transverse velocity $\left\langle v_{y}^{2}\right\rangle$ $=T_{y}$ is shown as a function of $y$ for three pipe flows in (e) and for $\beta>0^{\circ}$ flows in (f). Note that in (b), (d), and (f) the rescaled coordinate $y / w(x)$ is used on the horizontal axis.

the case and therefore we conclude that there is an important shear component to the flow.

The $y$ dependence of the $y$ component of the granular temperature, $T_{y}:=\left\langle v_{y}^{2}\right\rangle$, is shown in Fig. 9(e) for pipe flow and in Fig. 9(f) for $\beta>0^{\circ}$ flow. For the $D=15 \mathrm{~mm}$ pipe flow, $T_{y}(y)$ is constant while the wider pipe flows exhibit a more quiet region in the center-perhaps consistent with the "transverse momentum sink" mentioned in (ii) above. The $\beta>0^{\circ}$ flows in Fig. 9(f) are denser and slower, showing that in dense flows $\left(\beta=1^{\circ}, \beta=2^{\circ}\right)$ transverse momentum is absorbed immediately and the profile is flat. The "transverse heat sink" does not play a role here.

\section{POLYDISPERSE FLOWS}

To suppress the close packing effects that occur for monodisperse balls rolling in smooth funnels, we have also explored flows of balls of mixed sizes. We have studied shock waves for four different mixtures, which we will refer to as Mixture I-IV in increasing order of "polydispersity" (see Table I).

A crucial and unexpected effect of polydispersity can be seen in the space-time plots of the density such as Fig. 10. For a weakly polydisperse mixture [Mix I in Fig. 10(a)] the
TABLE I. Quantities of balls of various sizes in the four different polydisperse mixtures used here.

\begin{tabular}{cccccc}
\hline \hline Mixture & $2.5 \mathrm{~mm}$ & $3.0 \mathrm{~mm}$ & $3.2 \mathrm{~mm}$ & $3.5 \mathrm{~mm}$ & $4.0 \mathrm{~mm}$ \\
\hline I & & 10000 & 10000 & & \\
II & & 10000 & 10000 & 5000 & \\
III & 10000 & 10000 & 10000 & & \\
IV & & 10000 & 10000 & & 3000 \\
\hline \hline
\end{tabular}

shocks appear to display similar behavior as for monodisperse balls, but for increasingly polydisperse mixtures, shock waves lead to the blocking of finite fractions of the funnel. Here all balls are stationary for a finite time interval. Some clear examples of this "freezing" can be seen for Mix IV in Fig. 10(b), for $x \approx 80 \mathrm{~cm}$ and $t \approx 3.5 \mathrm{~s}$. The occurrence of finite blocked fractions can be interpreted in terms of a competition between the leading and trailing edge of a shock. For strongly polydisperse mixtures, the velocity of the trailing edge of the shock where the shocks dissolve, is lower than that of the leading edge where the shock grows due to incoming balls. As a function of its lifetime, a shock will therefore spread out, and finite regions of the funnel will be blocked. In contrast, when one would generate an extended blocked area in a monodisperse flows, it appears that the velocity of the trailing edge is larger than that of the leading edge. Such a shock would then shrink, until leading and trailing edge come very close together and the shock loses its spatial extent.

We have measured, employing ball tracking methods, the velocities of individual balls in shocks, and some representative results are shown in Fig. 11(a). The ball tracking method has not been specifically fine tuned for polydisperse flow, which leads to a slightly noisier determination of $v_{x}$. The overall picture that emerges is that in monodisperse flows, balls almost never stop completely in a shock [notice that for the example in Fig. 11(a) the minimum velocity of the balls in monodisperse shocks indeed stays finite]. In Mix I, complete stopping of balls in a shock occurs only in a
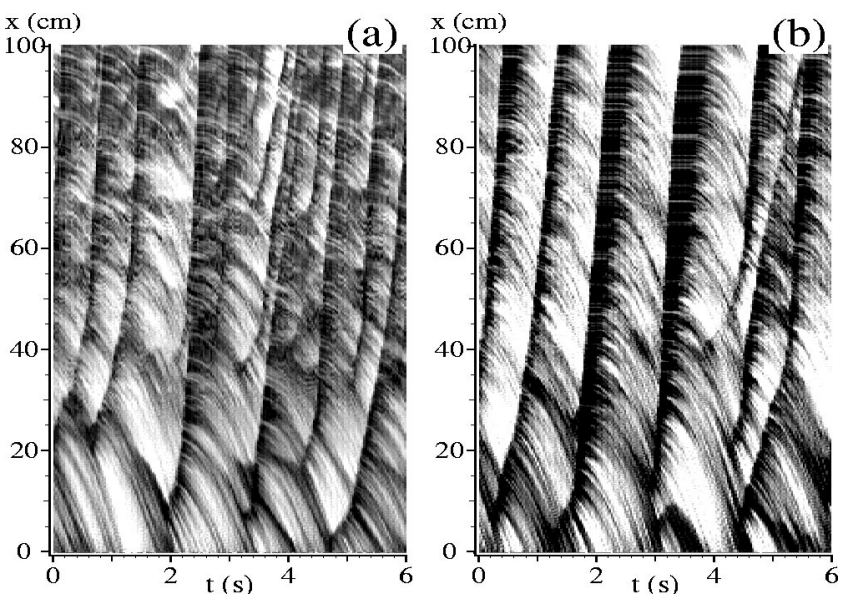

FIG. 10. Space-time diagrams of the density $\tilde{\rho}(x, t)$ showing differences in shock behavior between weak and strong polydispersity at $\beta=0.5^{\circ}, D=10 \mathrm{~mm}$ for Mix I (a) and Mix IV (b). 

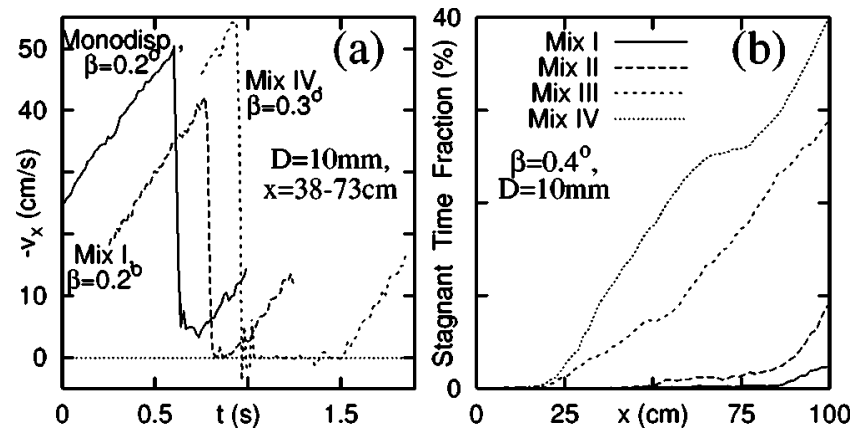

FIG. 11. (a) The $-v_{x}(\mathrm{t})$ of individual balls during the passing of a shock. (b) Fraction of time the flow stands still behind a just passed shock, based on density map data for polydisperse Mixtures I,II,III,IV.

minority of the shocks, but its occurrence increases with polydispersity and $x$. In Mix III complete stopping of balls occurs in most shocks that have propagated for more than $20-40 \mathrm{~cm}$ and do not have another shock right in front of them and in Mix IV blocking occurs essentially in all shocks.

To quantify this behavior further, we have measured (based on density space-time diagrams), for fixed values of $\beta$ and $D$ the fraction of time that the balls are stuck in such a stationary shock as a function of $x$ [see Fig. 11(b)]. This data confirms what we already observed in Fig. 10: the amount of blockage increases both with $x$ and with the strength of the polydispersity. The increase with $x$ can be understood simply from the observation that for strongly polydisperse mixtures shocks spread out during their lifespan, and since shocks travel upwards, the amount of blocked channel grows with $x$.

One possible explanation we can find for the increase of this blocking with polydispersity is the occurrence of 3D effects. One can imagine that in a shock wave, bigger balls that are squeezed between small balls are lifted from the support on which they roll. When such shock dissolves, small balls have to move over a finite distance before the bigger balls can start to roll, leading to a finite blocking time.

\section{Shock creation}

The polydispersity has two effects on the shock creation. One could have anticipated that for stronger polydispersity the periodic packing sites become irrelevant, but as we see from Fig. 12, what happens in addition is that all shocks are generated near the outflow of the funnel, an effect that can also be observed when comparing the density space-time diagram Fig. 10.

\section{Breakdown of scaling of $U(x)$ and $\nu(x) D$}

Using the RGE method we have studied to what extend the scaling of $U(x)$ and $\nu(x) D$ with $w(x) / D$ holds. In Fig. 13(a,b), we have plotted $U(x)$ and $\nu(x)$ for $\beta=0.4^{\circ}$ and $D$ $=10 \mathrm{~mm}$ for the four polydisperse mixtures. For Mix I, both $U(x)$ and $\nu(x)$ grow fairly linearly with the usual packing site related variations [5]. For Mix II the behavior is similar but both $U(x)$ and $\nu(x)$ become flatter for large $x$. For the stronger polydisperse Mixtures III and IV strong deviations from the monodisperse or weakly polydisperse case are ob-

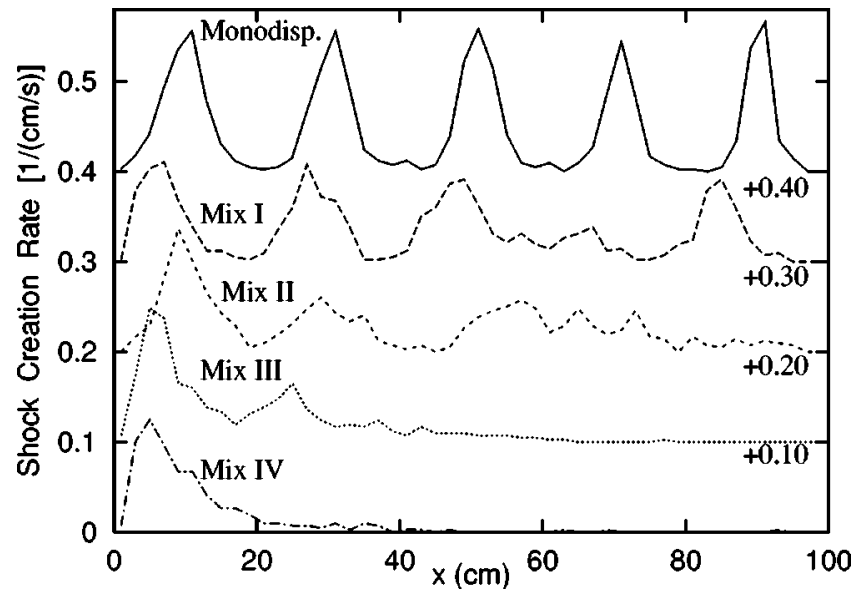

FIG. 12. Shock creation rates for monodisperse flow and polydisperse Mix I,II,III,IV. for $\beta=0.4^{\circ}, D=10 \mathrm{~mm}$.

served. The shock velocity $U(x)$ grows rapidly for small $x$, then peaks and subsequently begins to drop for larger $x$. The shock frequencies $\nu(x)$ in Mix III and IV behave similar to that of monodisperse or weakly polydisperse flows at small $x$ but become constant at larger $x$. This is fully consistent with
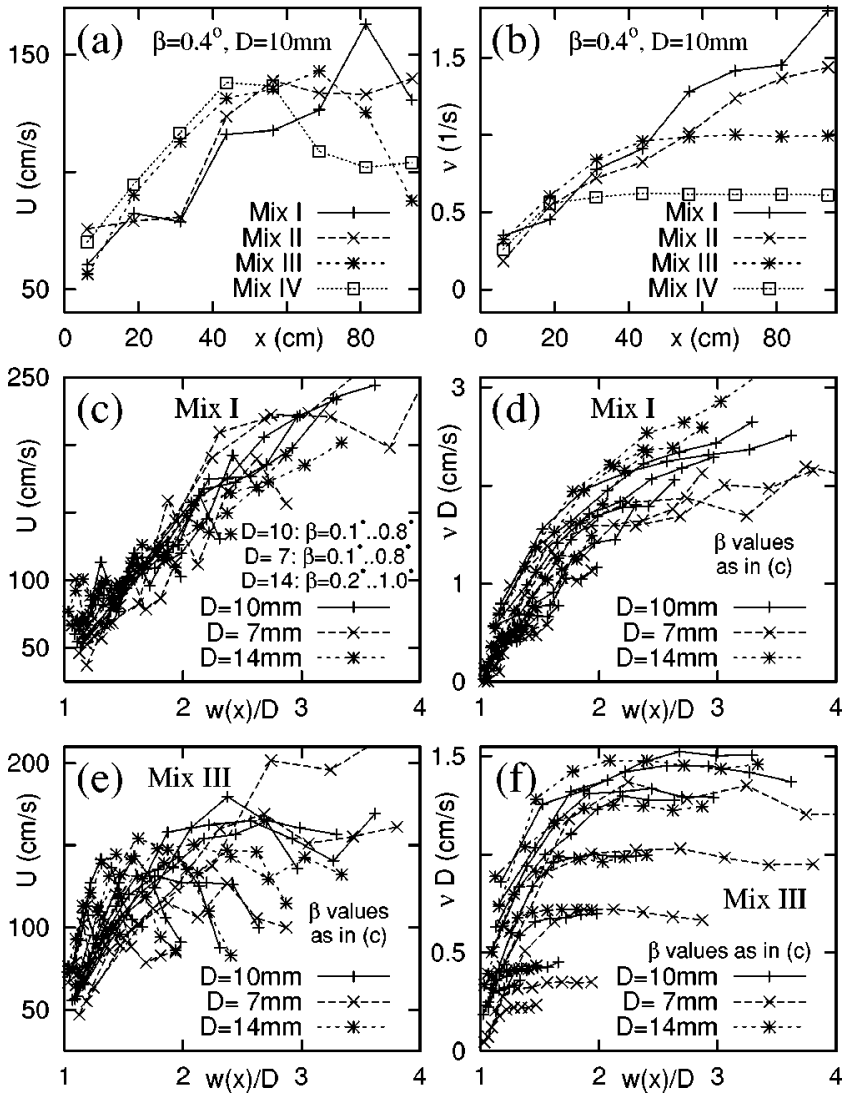

FIG. 13. RGE based plots of $U(x)(\mathrm{a}, \mathrm{c}, \mathrm{e})$ and $\nu(x)(\mathrm{b}, \mathrm{d}, \mathrm{f})$ : (a) shows $U(x)$ vs $x$ at $\beta=0.4^{\circ}, D=10 \mathrm{~mm}$ for Mix I-IV. (b) shows $\nu(x)$ vs $x$ at $\beta=0.4^{\circ}, D=10 \mathrm{~mm}$ for Mix I-IV. (c) shows $U(x)$ vs. $w(x) / D$ for Mix I. (d) shows $\nu(x) D$ vs $w(x) / D$ for Mix I. (e) shows $U(x)$ vs $w(x) / D$ for Mix III. (f) shows $\nu(x) D$ vs $w(x) / D$ for Mix III. [In each of (c-f) 18 data sets are displayed.] 
the data in Fig. 12 that shows that for strong polydisperse mixtures, all shocks are created near the outlet.

Based on the similarity between weak polydisperse and monodisperse flows we plot $U(x)$ vs $w(x) / D$ for Mix I in Fig. 13(c). Apart from packing site variations the data collapse is reasonably good, so in this respect the weakly polydisperse flow behaves as a monodisperse flow. Linear fits of the data in Fig. 13(c) yield $U(x) \approx 74[w(x) / D$ $-0.18] \mathrm{cm} / \mathrm{s}$. It is perhaps surprising that the mix I shock velocities are higher than those found for smooth wall monodisperse flows (see Sec. II A).

The frequency deserves some more attention. When we plot $\nu(x) D$ vs $w(x) / D$ in Fig. 13(d) the data collapse is not very convincing, especially for large $x$ where the rescaled frequency is widely spread. This may be due to beginning effects of polydispersity [causing $\nu$ to drop for high $x$ as shown in Fig. 13(b)] or partial failure of the RGE algorithm. This algorithm tends to discard weak shocks at high $\beta, x$ since the density contrast of the shocks becomes very small there (see Refs. [5,11]). Since $D=7 \mathrm{~mm}$ data (highest shock frequency and lowest density contrast) show the biggest deviation the latter reason may be the most important.

Despite the deviations for high $x$ there seems to be a data collapse of $\nu(x) D$ vs $w(x) / D$ which is superior to $\nu(x)$ vs $w(x) / D$. Together with the reanalysis of the older data [5] discussed in Sec. II A 3 this lead us to believe that the scaling of $\nu(x) D$ with $w(x) / D$ is a feature of both rough wall and smooth wall flows. Power law fits of the data in Fig. $13(\mathrm{~d})$ yield $\nu(x) D \approx 1.6(1)[w(x) / D-1]^{0.7(1)} \mathrm{cm} / \mathrm{s}$. That the shock frequencies of Mix I are slightly lower than for monodisperse flows is not surprising, since polydispersity generally seems to make it harder for shock waves to form anywhere else than near the outflow of the funnel.

For the $U(x), \nu(x)$ statistics based on Mix III density data shown in Fig. 13(e,f), there is data collapse for neither $U(x)$ nor $\nu(x) D$ vs $w(x) / D$. All $U(x)$ curves in Fig. 13(e,f) show the same pattern of rapid growth towards a maximum value after which a moderate decline sets in. We have found no clear pattern in the $x$ and $U$ values of the peaks, and there seems to be no $w(x) / D$ scaling involved. The $\nu(x) D$ data for Mix III shown in Fig. 13(f) displays growth at low $x$ followed by a plateau, but there is no clear trend in this plateau value. It seems likely that both types of deviation from the monodisperse scaling relations are linked to the stagnant regions behind shocks and thus to the threedimensional packing effects discussed above.

The $U(x)$ and $\nu(x)$ data for polydisperse Mix II (not shown) are similar more to Mix I, while the Mix IV data (not shown) are similar to the Mix III data.

In conclusion, we find that for sufficiently strong polydispersity, the nature of the shock waves changes qualitatively, and that scaling relations that hold for monodisperse flows in either smooth or rough funnels break down.

\section{DISCUSSION}

This work, in combination with earlier work on smoothwall-monodisperse flows [5,6], leads to a number of conclusions about the effects of funnel geometry, wall roughness and inelastic dissipation. It is well known that the dissipation occurring in ball-ball and ball-wall collisions is enhanced by the rolling nature of the ball motion [14]; however, the precise value of the effective coefficient of restitution is presumably not of large importance for the phenomenology. In particular, the frequency and velocity scaling for monodisperse and weakly polydisperse systems are very similar (see Fig. 7), even though for weakly polydisperse flows dissipation seems enhanced. For strongly polydisperse flow, where dissipations seems very strong, we unfortunately lose the $2 \mathrm{D}$ nature of the experiment.

In the case of smooth walls and monodisperse flows, packing effects become very important, and they tend to obscure scaling law. Possibly the simplest case is then the combination of rough walls and monodisperse flows (or, to a lesser degree, smooth walls and weakly polydisperse flows). The rough walls have the additional advantage that they make the system less sensitive to small perturbations (which are inevitably present for "smooth" walls).

The scaling laws for shock velocity and frequency, Eqs. (2) and (3), are the main result of our work. Since these laws also approximately hold for smooth walls, etc., their main origin must lie in the geometry of the experiment. That the shock velocity and frequency depend on $x$ via $w(x) / D$ is not surprising, since this is the most obvious way in which $x$ can be made nondimensional (we do not expect the ball diameter to play an important role). The fact that $\nu(x) D$ and not $\nu$ scales is harder to understand, and may point at certain relevant velocity scales (note that both $U$ and $\nu D$ have the dimension of velocity). Two-dimensional quantities that characterize the system are the effective ball-acceleration $a_{\text {eff }}$ (related to the inclination) and $D$, but these two quantities alone are not sufficient to provide for the correct scaling factors, since a velocity scale would be $\sqrt{D a_{\text {eff }}}$, and a frequency scale would be $\sqrt{a_{\text {eff }} / D}$, which both show scaling with $D$ different from what is observed.

We believe that the underlying reason for the occurrence of the scaling laws is an important open question that deserves further study. Our data indicates that details of the ball-ball or ball-wall interactions are not important (although rough walls lead to more frequent shocks, they do not alter the nature of the scaling), which suggests that a relatively straightforward model may capture the phenomenology here. Indeed, for pipeflow, where the geometrical cause of shock formation dissapears, leads to quite fragile behavior, which only in the case of rough walls seems to reproducable and similar to small funnel angle behavior.

However, to make such a model one seems to need additional information about the relation between ball densities and velocities on one hand, and shock frequencies and velocities on the other.

Finally, shock waves have also been studied in some other geometries. Tsai et al. [7] examined a two-dimensional rolling pipe flow of $3.2 \mathrm{~mm}$ steel spheres between rough walls. Partial blockage of the funnel outlet was used as an additional system parameter. These authors performed high resolution ball tracking measurements of small $(12 \mathrm{~cm})$ sections of the funnel and obtained detailed local measurements of 
$v(x, t), \tilde{\rho}(x, t), T_{y}(x, t)$, and $v_{x}(y)$. For an almost unblocked flow with $D=19 \mathrm{~mm}$ they found $\nu \approx 0.41 / \mathrm{s}$ and $U$ $\approx 14 \mathrm{~cm} / \mathrm{s}$, which is consistent with our data.

Reydellet, Rioual, and Clément [8] studied $1.5 \mathrm{~mm}$ metallic balls in a vertical pipe flow with rough walls. The balls were not rolling on a support, and the rolling of balls supposedly played less of a role than in our experiments. They qualitatively observed the existence of upwards propagating shock waves, using a "double flash technique" to study local ball velocities and made measurements similar to those in our Fig. 9. Note that their funnel was not continuously refilled from a reservoir and thus their studies were limited to the transient behavior. All our experiments are preceded by a $>30 \mathrm{sec}$. flow in order to avoid such transient behaviors.

Finally, Le Pennec et al. [9] studied a two-dimensional rolling flow of $1 \mathrm{~mm}$ glass balls in flow geometries with very large $\beta$ (mostly $30^{\circ}$ ), large $D$ (typically $10-120$ ball diameters) at various flow plane inclinations. Due to the different geometry it is hard to make any direct comparison, although these authors did measure shock velocities and frequencies.

\section{SUMMARY}

The packing effects that we observed in earlier work on monodisperse, smooth-walled flow [5,6] can be suppressed by either making the side walls rough or using polydisperse mixtures of balls. For rough walls, we find that there are more shock waves than for smooth walls as a result of the greater disturbances in this flow. In earlier work [5] on smooth-wall flows we found that the average shock speed $U(x)$ scales with $w(x) / D$. We have found a similar scaling for rough walls and observed that also $\nu(x) D$ scales with $w(x) / D$ for both rough and smooth walls [12]. By using ball tracking methods we shed light on the shear flow properties for rough walled flows.

In polydisperse flows between smooth walls we find that our scaling relations persist for very weak polydispersity, but breaks down for stronger polydispersity. This breakdown is most likely caused by weak three-dimensional effects in the packing of shock waves, which apparently lead to partial blocking of the funnel. By using ball tracking methods we shed light on the shock structure and on the extended stationary shock packings found in strong polydisperse flows.

\section{ACKNOWLEDGMENTS}

The authors wish to thank Christian Veje for making the original rough walls. P.D. would like to thank Statens Naturvidenskabelige Forskningsråd (Danish Research Council) for support. M.v.H. would like to thank CATS for continuing hospitality.

\section{APPENDIX A: COMPARISON WITH TRAFFIC FLOW}

To compare our flows to traffic flows, small angle, or preferably pipe flows should be considered. In earlier work we made comparisons between smooth-wall funnel flows with nonzero $\beta$ and traffic flows [6]. In smooth-wall pipe flows, shock waves are extremely fragile, and such a com-
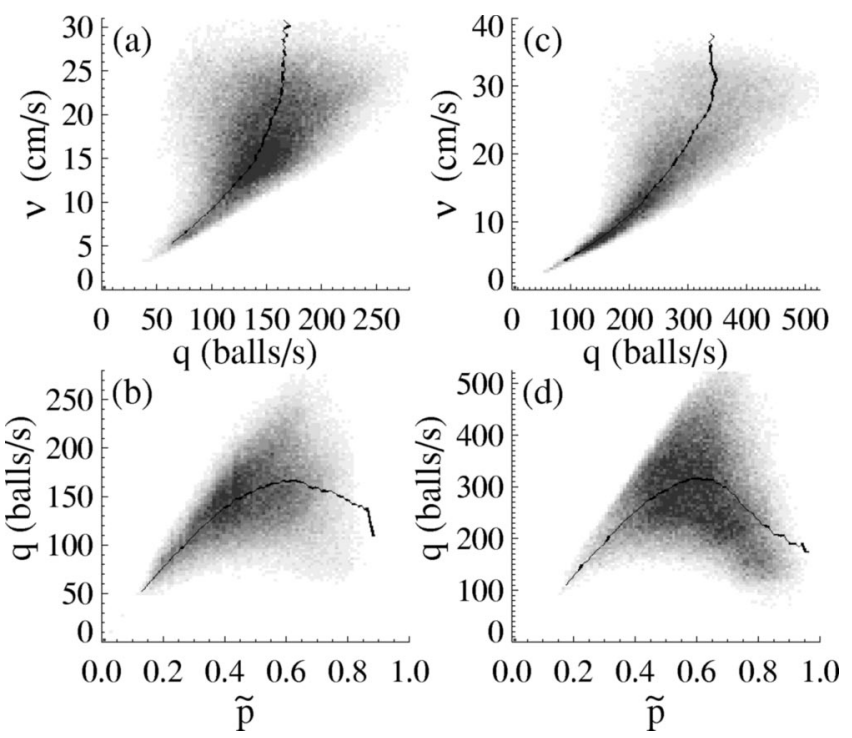

FIG. 14. Fundamental diagrams in rough wall flows. (a) and (b) Pipe flow studied in the interval $37<x<74 \mathrm{~cm}$ for $\beta=0.0^{\circ}$ and $D=15 \mathrm{~mm}$. (c) and (d) Intermittent flow studied in the interval 0 $<x<37 \mathrm{~cm}$ for $\beta=0.4^{\circ}$ and $D=20 \mathrm{~mm}$.

parison is not meaningful. For the rough wall pipe flow discussed here, however, such a comparison can be made in principle. In addition, we will discuss our results for rough wall funnel flows in terms of traffic flows.

It is commonly assumed that there are three flow types in traffic flow, namely, uncongested flow (a steady flow of vehicles at low to moderate densities, queue flow (a slow flow of near maximum density), and queue discharge (a flow of vehicles accelerating out of a queue flow) [10]. Traffic data are usually represented as $v(q)$ (speed/flow relation) or as $q(\rho)$ (fundamental diagram), where $q=\rho v$ is the flow rate.

In Figs. 14(a) and 14(b) we show gray scale histograms of $(q, v)$ and $(\tilde{\rho}, q)$ for a rough wall pipe flow $\left(\beta=0^{\circ}, D\right.$ $=15 \mathrm{~mm})$. Note that we use the relative density $\tilde{\rho}$ instead of $\rho$. The average values, corresponding to $v(q)$ and $q(\tilde{\rho})$, are shown as solid lines. In Fig. 14(a), we observe regions corresponding to queue flow and queue discharge, but there is no region corresponding to uncongested flow. As shown in Fig. 14(b), the flow rate $q(\tilde{\rho})$ has a parabolic shape with a peak value around $\tilde{\rho}=0.6$.

For larger values of $D$ (not shown), the flow rates exhibit similar parabolic behavior, albeit with larger typical values of $v$ and $q$, and with the $q(\tilde{\rho})$ "parabola" skewed towards lower $\tilde{\rho}(\tilde{\rho}=0.4-0.5$ for $D=20 \mathrm{~mm}, \tilde{\rho}=0.2-0.4$ for $D$ $=25 \mathrm{~mm}$ ).

Figures 14(c) and 14(d) show the corresponding diagrams for small angle, rough wall funnel flow at $\beta=0.4^{\circ}, D$ $=20 \mathrm{~mm}$. We find queue flow and queue discharge but no uncongested flow, similar to what we found in earlier work on smooth-wall funnel flow [6]. In Fig. 14 we observe a parabolic shaped $q(\tilde{\rho})$. Similar plots for higher $\beta$ (not shown) exhibit "parabolas" skewed towards higher values of $\tilde{\rho}$. 


\section{APPENDIX B: VELOCITY AND FREQUENCY DETERMINATION FOR PIPE FLOW}

The method to obtain shock velocities for pipe flow data is illustrated in Fig. 15. First of all, to highlight the shocks that occur in the density plots, some smoothing and differentiation is applied yielding a field $s(x, t)$ as shown in Fig. 15(a). Clearly, shocks are now visible as bright streaks (high values of $s$ ) in a fairly even background. The temporal averages of the spatiotemporal correlation function $C(x, \Delta x, \Delta t):=\int d t s(x, t) s(x+\Delta x, t+\Delta t)$, two examples of which are shown in Figs. 15(b) and 15(c) for $x=130$ and 170 $\mathrm{cm}$, clearly show a dominant direction in space-time that can be associated with the local dominant velocity of the shocks: this method of shock velocity determination works over the whole range of parameters considered. In comparison to the RGE method, this method is more local, and often gives a slightly smaller estimate for the velocities (order of 10$20 \%$ ). This is presumably due to the fact that shocks have a tendency to perform intermittent jumps forward, making their dominant local velocity smaller than the typical velocity obtained over longer timescales. Some effect of these jumps shows up in the correlation functions, where the bright streak tends to bend for larger correlation distances and time intervals.
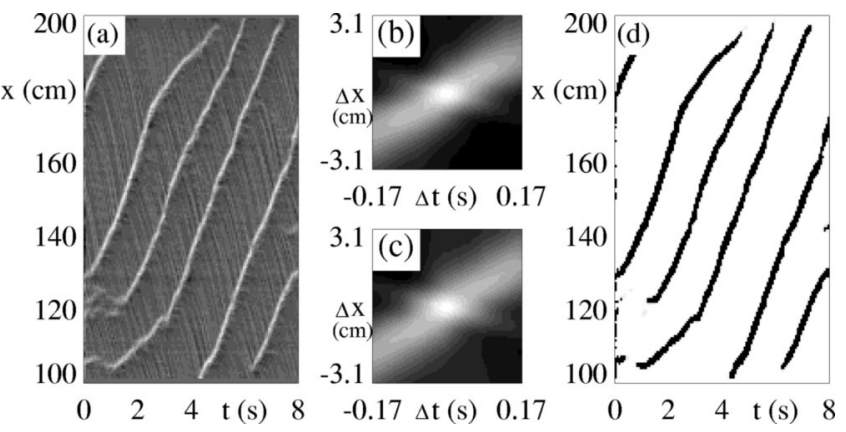

FIG. 15. Illustration of methods used to obtain shock velocities and frequencies for pipe flow for the case of $D=20 \mathrm{~mm}$. (a) Modified density plot. (b) and (c) Space-time correlation functions based on data shown in (a), for (b) $x=130 \mathrm{~cm}$, and (c) $x=170 \mathrm{~cm}$. Note that the scales of (a), (b), and (c) are different, so that the dominant angles appear different. (d) Thresholded data used for determination of shock frequency.

To obtain the frequency from $s(x, t)$ is fairly straightforward: after choosing an appropriate threshold, one obtains two-color images as shown in Fig. 15(d). A simple algorithm suffices to count, for a fixed value of $x$, the number of shock waves that occur.
[1] R.L. Brown and J.C. Richards, Principles of Powder Mechanics (Pergamon Press, Oxford, 1970); J. Lee, S.C. Cowin, and J.S. Templeton, Trans. Soc. Rheol. 18, 247 (1974); R.L. Michalowski, Powder Technol. 39, 29 (1984); G.W. Baxter, R.P. Behringer, T. Fagert, and G.A. Johnson, Phys. Rev. Lett. 62, 2825 (1989); A.A. Mills, S. Day, and S. Parkes, Eur. J. Phys. 17, 97 (1996); also, in Pattern Formation and Complexity in Granular Flows, edited by R.P. Behringer and G.W. Baxter (Springer-Verlag, Berlin, 1993).

[2] T. Pöschel, J. Phys. I 4, 499 (1994); S. Horikawa, A. Nakahara, T. Nakayama, and M. Matsushita, J. Phys. Soc. Jpn. 64, 1870 (1995); T. Raafat, J.P. Hulin, and H.J. Herrmann, Phys. Rev. E 53, 4345 (1996); O. Moriyama, N. Kuroiwa, M. Matsushita, and H. Hayakawa, Phys. Rev. Lett. 80, 2833 (1998).

[3] K.L. Schick and A.A. Verveen, Nature (London) 251, 599 (1974); X.-1. Wu, K.J. Måløy, A. Hansen, M. Ammi, and D. Bideau, Phys. Rev. Lett. 71, 1363 (1993); C.T. Veje and P. Dimon, Phys. Rev. E 56, 4376 (1997); C.T. Veje, Ph.D. thesis, University of Copenhagen, 1999.

[4] C.T. Veje and P. Dimon, Phys. Rev. E 54, 4329 (1996); C.T. Veje, Master's thesis, University of Copenhagen 1995.

[5] S. Hørlück and P. Dimon, Phys. Rev. E 60, 671 (1999); S. Horluck, Master's thesis, University of Copenhagen, 1997.

[6] S. Hørlück and P. Dimon, Phys. Rev. E 63, 031301 (2001).

[7] J.-C. Tsai, W. Losert, G.A. Voth, and J.P. Gollub, Phys. Rev. E 65, 011306 (2001).

[8] G. Reydellet, F. Rioual, and E. Clément, Europhys. Lett. 51, p.27 (2000).
[9] T. Le Pennec, M. Ammi, J.C. Messager, and A. Valance, Eur. Phys. J. B 7, 657 (1999).

[10] See, for example, in Workshop on Traffic and Granular Flow, edited by D.E. Wolf, M. Schrenkenberg, and A. Bachem (World Scientific, Singapore, 1995).

[11] The shock detection algorithm RGE (Relative density contrast Gradient Edge detection) used on $\tilde{\rho}$ data is a refinement of the GE method described in Ref. [5]. It is used to measure $U(x)$ and $\nu(x)$. We use two versions: RGE4 with an $x$ res. of $25 \mathrm{~cm}$ and RGE8 with an $x$ resolution of $12.5 \mathrm{~cm}$. The RGE algorithm gives more reliable measurements of average shock frequency $\nu(x)$ but may still underestimate it under conditions with high $\nu$ and very low $\tilde{\rho}$ contrast. The RGE method does not detect shocks with $U<15-20 \mathrm{~cm} / \mathrm{s}$ as reliably. This is only a real problem in rough walled pipe flows.

[12] This was not shown in Ref. [5] but with improved data analysis [11] of the old data we find a decent data collapse when plotting $\nu(x) D$ vs $w(x) / D$.

[13] The shock creation rates shown here have been hand counted, and every shock that propagates upwards at least $5 \mathrm{~cm}$ (no matter its strength or continued existence) is counted. The shock wave creation position statistics shown in Fig. 18 in Ref. [5] were based on a image processing method (DT-no longer used), that only counted shocks of a certain strength. Consequently this method showed a decreasing shock creation rate with increasing $w(x)$ (for smooth walls).

[14] B. Painter and R.P. Behringer, Phys. Rev. E 62, 2380 (2000). 